\title{
Renouveler les modèles de la communication et de la coopération par l'improvisation : Org'impro, une recherche-action innovante
}

Valérie Lépine, maître de conférence, Université Grenoble Alpes, laboratoire Gresec, valerie.lepine@univ-grenoble-alpes.fr

Fabienne Martin-Juchat, professeur, Université Grenoble Alpes, laboratoire Gresec, Fabienne.martin-juchat@univ-grenoble-alpes.fr Thierry Ménissier, Professeur, Université Grenoble Alpes, Laboratoire Philosophie, Pratiques \& Langages

Thierry.Menissier@univ-grenoble-alpes.fr 


\title{
Résumé
}

Cette contribution s'inscrit dans la continuité des recherches qui questionnent la formation en compétences communicationnelles des futurs cadres (professionnels de la communication et managers). Elle met en évidence la place du non verbal dans l'élaboration du faire ensemble et interroge les modèles enseignés sur le rôle de ce type de communication dans la construction d'un projet collectif. Par le compterendu et l'analyse d'une proposition pédagogique originale intitulée « Org'impro », recherche-action dans le domaine de la formation ayant impliqué des chorégraphes spécialistes de l'improvisation et portée par l'Université Grenoble Alpes, notre propos est de nourrir la pédagogie par la recherche et de développer des dispositifs de formation transférables dans les organisations.

Mots-clefs : communication non verbale, improvisation, compétences, coopération.

\begin{abstract}
This contribution takes place into the field of researches that question the communication skills training for future managers (communication professionals and managers). It highlights importance of nonverbal communication in cooperative work and questions the models taught about the role of this type of communication for building a collective project. By the report and analysis of an original educational proposal entitled "Org'impro ", an action research in the field of training that involved specialists choreographers of improvisation and managed by the University of Grenoble Alpes, our purpose is to increase the pedagogy research and develop training devices transferable in organizations.
\end{abstract}

Keywords: non verbal communication, improvisation, skills, cooperation. 
Cette contribution vise à rendre compte et à analyser une recherche-action dans le domaine de la formation intitulée Org'impro, menée en janvier 2015 dans le cadre du programme Promising de l'université Grenoble Alpes ${ }^{88}$ et visant à expérimenter une pratique pédagogique innovante. Cette pratique, par sa portée critique et réflexive, interroge les modèles de la communication et du management enseignés.

L'équipe de conception du dispositif était composée de deux enseignantschercheurs et de deux artistes chorégraphes ${ }^{89}$ : les universitaires Fabienne MartinJuchat, spécialiste de la communication non-verbale dans les organisations et anthropologue du mouvement (Martin-Juchat, 2008, 2014 ; Lépine, Martin-Juchat \& Millet-Fourrier, 2014) et Thierry Ménissier, spécialiste de philosophie politique et d'anthropologie de l'action collective (Ménissier, 1999, 2003, 2011) et les deux chorégraphes-improvisateurs François Veyrunes et Emmanuel Grivet. Responsables de compagnies et formateurs de danseurs, ces artistes interviennent également dans d'autres secteurs et avec des publics de non-danseurs. L'intention initiale de ce collectif était de déterminer puis de faire pratiquer des exercices permettant de faire expérimenter aux participants différentes étapes du faire ensemble : l'écoute de soi et des autres dans un lieu donné ; la perception de soi et des autres dans l'espace ; la manière dont on entre dans un espace, selon une séquence marquée par un début, un terme et une vitesse de déplacement; la manière dont on chemine pour inscrire un acte en relation avec celui d'autrui ; le choix de l'acte, qu'il s'opère en complément, en contre-point, en opposition ou en répétition. En d'autres termes, les exercices choisis visaient à faire ressentir les grammaires de l'action et de l'interaction les plus courantes (présence, co-présence, coordination par addition, par subordination, coopération).

Ont participé à l'expérimentation une quarantaine d'étudiants inscrits en masters de sciences de l'information et de la communication et de management de l'innovation, des doctorants issus de diverses disciplines académiques, une demidouzaine d'enseignants-chercheurs et une dizaine de personnes pratiquant l'improvisation et informées par le partenaire du dispositif, l'association CitéDanse de Grenoble. Les chercheurs se sont répartis en deux groupes : observateurs (Aurélia Dumas, Fabienne Martin-Juchat et Thierry Ménissier) et en immersion (Valérie Lépine, Muriel Guigou et Caroline Angé).

Le protocole a reposé sur une phase théorique portant sur la manière dont est pensé le non verbal dans les organisations et une phase de préparation pratique de deux fois deux heures d'initiation à l'improvisation (réalisée par Muriel Guigou et

\footnotetext{
${ }^{88}$ Cette recherche a bénéficié d'un financement du programme Promising, IDEFI n ANR-11IDEFI-0031

${ }_{89}$ D'un point de vue méthodologique, nous insistons sur la nécessité d'une véritable coconstruction avec les artistes du dispositif (pour plus de détails sur l'enjeu de ce type de méthode lire : Martin-Juchat, Zammouri, 2013).
} 
Etienne Quintens, respectivement sociologue et pédagogue de la danse), suivie d'une journée séquencée de la sorte : une matinée d'ateliers d'immersion et d'apprentissage des principes et techniques de l'improvisation (animés par les deux artistes chorégraphes) et une après-midi de travail en groupes restreints visant l'appropriation et la mobilisation des acquis par la création d'une composition collective, puis retour d'expérience final en assemblée plénière. Les ateliers du matin ont alterné des exercices en grand groupe (une vingtaine de participants) et des improvisations basées sur la construction de tableaux vivants par petits groupes (dix personnes observaient pendant que dix autres intervenaient). Les observateurs appréhendaient les différentes formes de la construction du faire ensemble. Le principe pédagogique était de ressentir les étapes d'élaboration d'un projet par un collectif improvisé dans l'espace. Les participants entraient dans l'espace un par un à partir des consignes suivantes: attendre qu'un participant se soit positionné, observer les tactiques de mises en place (ajouts par symétrie, complément, opposition, répétition), puis prendre place soi-même, à son rythme, dans une position libre. Le jeu permettait de goûter la manière dont les autres composent ou non avec une proposition et de s'observer en train de prendre une position de leader ou de suiveur. La matinée a donc été consacrée à la transmission de techniques, afin de permettre aux participants de se familiariser avec la composition instantanée, entendue comme capacité à produire un acte en s'inscrivant dans un contexte donné. L'après-midi, des groupes différents ont été constitués et un événement organisé afin d'activer de la coopération: chacun des groupes a préparé son propre projet d'improvisation et de composition en choisissant des règles d'organisation, en vue d'une performance finale : une scénette de quelques minutes soumise au regard des autres groupes. Cette phase conférait une grande autonomie aux participants libres de s'appuyer sur les techniques découvertes le matin ou d'inventer leur propre vocabulaire chorégraphique, les ateliers ayant été présentés comme des espaces où il est possible de concevoir liberté et créativité en complémentarité dans l'optique de faire ensemble.

Suite à cette expérimentation, deux types de données ont été collectés : un corpus de travaux écrits produits par l'ensemble des étudiants dans le mois suivant la journée pédagogique (40 rendus) ${ }^{90}$ et un corpus d'entretiens individuels récoltés

\footnotetext{
90 Les questions posées étaient les suivantes: Pour quelles raisons l'expérience corporelle et affective a-t-elle toujours été un enjeu sociopolitique et socio-économique ?; En quoi Org'Impro est-il un événement révélateur de ces raisons et/ou tendances et/ou attentes ?; Quels liens entre improviser et les conditions du travail collectif?; En quoi improviser apprend-il à construire ensemble ?; Avez-vous testé des comportements dont vous n'avez pas l'habitude ?; Qu'avez-vous appris sur le leadership ?; Vous êtes-vous découvert des compétences, habilitées grâce à cette journée ? Comment la mise en mots se pose face à l'improvisation et réciproquement? Qu'avezvous le plus et le moins apprécié ?
} 
auprès des participants $\left(15\right.$ entretiens $\left.{ }^{91}\right)$, volontairement trois mois après l'expérimentation pour obtenir des témoignages sur ce qui a fait trace, avec le postulat qu'un temps s'imposait afin que les participants puissent mettre des mots sur l'expérience vécue ${ }^{92}$. La question est de savoir en quoi l'improvisation peut à la fois fournir et donner à comprendre les conditions d'émergence du faire ensemble. L'hypothèse de base du dispositif est que mettre en situation d'improvisation des étudiants - futurs professionnels de la communication, de la médiation et du management - leur permet d'acquérir ou développer des compétences communicationnelles «invisibles». Ces compétences sont ainsi qualifiées car bien que relevant des conditions du faire ensemble, elles demeurent la plupart du temps minorées dans les objectifs des formations comme au sein des entreprises, véritables «dimensions oubliées» de l'être humain (Chanlat, 2005). Or, s'appuyer sur des techniques d'improvisation permet effectivement de prendre conscience, par l'expérimentation collective et dans l'expérience individuelle vécue, des conditions de construction d'un projet collectif et en particulier de la place du non verbal dans la relation à soi, aux autres et au groupe. Ce mode de construction d'une action collective improvisée permet de rendre saillantes les limites des modèles de management les plus couramment appris.

\section{1. Éléments de cadrage théorique : improvisation et compétences du faire ensemble}

L'improvisation par le mouvement fournit une connaissance originale ainsi que des outils de compréhension et des modes d'approche créatifs du faire ensemble. Elle révèle les dimensions invisibles, les variables et les étapes de la coordination et de la coopération, telles que : l'écoute, l'inter-influence, l'alternance de temps de prise de parole (acte de langage verbal ou non verbal) et de silence. Concernant le faire ensemble, il a permis de mettre en évidence la grammaire inhérente à une praxéologie : la coordination par apposition d'actes, la coopération faible - lorsqu'il s'agit de suivre une mise en ordre dans un processus déterminé - et la coopération forte - quand il faut agir en commun en partageant la signification de l'action engagée - telles que les a définies Zarifian (1996).

Notre hypothèse est que les conditions du succès du faire ensemble se situent en grande part dans le non verbal plutôt que dans le contenu des échanges produit lors

\footnotetext{
${ }^{91}$ Entretiens d'une heure environ, réalisés par Fabienne Martin-Juchat, Thierry Ménissier, Muriel Guigou et Anne Balthazar (participante et danseuse).

${ }^{92} \mathrm{La}$ trame de la grille d'entretien qualitative reposait sur plusieurs thèmes : retour distancié sur l'expérience, sur soi, sur le déroulé de la journée, sur les perspectives de transférabilité de l'expérience.
} 
des traditionnelles réunions au sein des organisations de travail, si bien que nombre de réunions s'avèrent décevantes du point de vue de la coopération attendue.

\title{
1.1. L'improvisation : éléments de définition
}

L'improvisation n'est ni la non-organisation, ni la non-pensée, mais une relation entre participants à l'action qui favorise la circulation entre différents types de mouvements : 1'action, l'interaction, l'émotion, la pensée (Martin-Juchat, 2008). Elle construit des passerelles entre des processus traversant le corps et l'esprit, en provenance non seulement des systèmes d'interactions au sein du groupe mais aussi présents dans l'environnement. Procédant par d'incessants ajustements que chacun met en œuvre de manière non consciente tant dans sa vie privée que dans un cadre professionnel en vue de composer avec d'autres, elle constitue une des conditions invisibles de la coopération. Dans le champ artistique, c'est une pratique précédemment théorisée :

\begin{abstract}
«L'improvisation ne signifie aucunement un laisser-aller, il faut se garder de considérer naïvement que l'improvisation, de son côté, ressortirait à un principe généralisé du "n'importe quoi". L’improvisation demande de mobiliser des grilles, des consignes ou des principes, préalablement posés. Elle requiert de connaître des techniques, notamment dans les registres sensoriels et relationnels, l'improvisateur devant se mettre à l'écoute, mobiliser des capacités de connexions, et finalement d'élaboration de ce qui est aussi appelé une composition instantanée » (Mayen, 2012).
\end{abstract}

L'improvisation crée une attitude dans le rapport à soi, aux autres et au monde qui permet de capter la connaissance tacite présente dans l'environnement puis de la mettre en formes. Elle construit des situations indéterminées qui s'apparentent à du jeu, ce qui facilite l'engagement et introduit de la souplesse et de la circulation entre des phases de mouvement réglées ou non, langagières ou non. $\mathrm{Ce}$ jeu construit un dialogue, un pont entre règles et créativité, ainsi qu'entre soi, les autres et le monde. Il favorise, de plus, le partage et la création de liens sociaux en conduisant à des mouvements partagés (des émotions, du latin emovere) qui favorisent l'émergence d'une communauté d'appartenance, même provisoire. Il permet la prise de risque dont a besoin l'individu pour s'extraire du poids des représentations et adapter son geste et sa pensée aux événements imprévus. Il peut, enfin, conduire à davantage de souplesse dans la gestion des rôles de suiveur ou de leader et nourrit un questionnement dans le rapport à ces rôles, en particulier ceux de celui qui orchestre, qui dirige ou qui sait. L'ouverture d'un tel espace nous paraît utile à la réflexivité des professionnels de la communication comme aux futurs managers et présente également un intérêt en termes d'émancipation. 


\subsection{Les compétences du faire ensemble}

La notion de compétence est polysémique : des définitions et les usages sociaux et professionnels variés ont émergé puis évolué dans des champs disciplinaires pluriels et selon des contextes nationaux, historiques ou organisationnels divers. Tous sont à resituer dans la perspective des modèles de gestion ou de formation qui s'y réfèrent (Dietrich, Gilbert, Pigeyre, 2010). Sans qu'on puisse parler d'un concept qui serait aujourd'hui consensuel ou stabilisé, il est admis que la compétence, dans un contexte professionnel, renvoie à un agir reconnu comme efficace face à un ensemble de situations ou de pratiques professionnelles. Elle constitue un « savoiragir socialement reconnu » (Wittorski, 1998) qui permet d'obtenir un résultat; elle est liée à une performance, une réalisation (qui peut être mentale, physique ou encore organisationnelle). La performance devient compétence lorsqu'elle est reconnue par un tiers dans l'environnement, « en rapport à une norme sociale d'activité » (Hillau, 1994). Des auteurs comme le sociologue Philippe Zarifian ou le praticien-chercheur Guy Le Boterf récusent les modèles statiques qui assimilent les compétences à un ensemble de ressources (traditionnellement regroupées en trois catégories dont la vertu principale est la simplicité : savoirs, savoir-faire et savoirêtre) pour souligner le caractère dynamique et processuel de la compétence. Zarifian (2010) la définit comme « la prise d'initiative et de responsabilité de l'individu sur des situations professionnelles auxquelles il est confronté » en situation. Le Boterf (2004) entend se défaire d'une approche qui substantialise ou isole les compétences - comme tendent à le faire les promoteurs des référentiels de compétences - pour prendre en compte « ce que signifie, pour un sujet, être compétent (...) c'est-à-dire être capable d'agir et de réussir avec compétence dans une situation de travail (...) en mobilisant une combinatoire appropriée de ressources » qui peuvent être cognitives, comportementales, procédurales, techniques ou socio-affectives. Ces auteurs mettent aussi l'accent sur l'importance de l'environnement qui rend possible la mobilisation de l'intelligence pratique, laquelle prend appui tant sur des ressources pratiques et organisationnelles que sur des réseaux d'acteurs, car il s'agit de nouer des coopérations sans lesquelles de nombreuses situations seraient impossibles à affronter.

La compétence s'inscrit aussi dans un processus identitaire (Ardouin, 2004) implicitement ou explicitement en référence à une "professionnalité », " composée de compétences, de capacités, de savoirs, de connaissances et d'identités reconnus par une organisation ou un groupe professionnel comme étant les caractéristiques d'un «vrai » professionnel » (Wittorski, 2008, p. 21). Ainsi appréhendée comme une construction sociale, elle procède d'une intention (par exemple celle de l'amélioration de l'efficacité des organisations ou de la productivité), d'une négociation pour obtenir une légitimité qui s'appuie sur la défense corporative d'une expertise. Pour les métiers de la communication, en France notamment, en évolution 
constante depuis l'apparition d'une fonction dédiée (Walter, 1995), la production de codes, chartes, référentiels de métiers ou de compétences révèle le caractère polyphonique de la représentation professionnelle associative et syndicale et témoigne d'une professionnalisation « inachevée » et en quête de reconnaissance (de la Broise, 2013). Les compétences très transversales indispensables aux métiers de la communication et de l'encadrement sont souvent convoquées sans être réellement spécifiées, et généralement peu prises en compte dans les formations, sinon sous la forme d'aptitudes secondaires liées aux travaux de groupe proposés aux étudiants. La place qui leur est faite dans les référentiels évoque une injonction à adopter des comportements coopératifs requis par le bon fonctionnement des activités et des projets, sans pour autant donner de consistance pratique et expérientielle à leur développement.

Enfin, les approches en psychologie didactique, telle celle proposée par Gérard Vergnaud (1990) pour qui il faut penser l'apprentissage en termes de processus de conceptualisation, ont fait progresser tant la réflexion que les pratiques pédagogiques et de didactique professionnelle en s'intéressant à la part mentale de construction des schèmes qui orientent l'action. La compétence est apprise au sens où des cognitions repérables sont mobilisées et possiblement modélisées. D’autres recherches en sciences cognitives ou en ergonomie portent leur attention à la part incarnée du travail, au savoir incorporé dans un geste métier. Dans son ouvrage consacré au "praticien réflexif», Donald Schön (1994) propose de mettre à la portée des professionnels l'explicitation des savoirs invisibles ou cachés qui se traduisent pourtant en agissements experts, en actes précis et adaptés aux situations.

En regard de ces éléments, puisque c'est la situation vécue qui révèle des compétences, Org'impro est à comprendre comme une mise en situations des participants, afin de leur permettre de mobiliser leur intelligence pratique. Celle-ci se manifeste dans des attitudes d'ajustement instantané aux mouvements et aux tableaux composés sans planification préalable, dans une capacité à lire et se nourrir de l'environnement tel qu'il se donne dans l'instant. Nous souscrivons, de plus, à l'idée que l'ancrage d'une compétence professionnelle, qui puisse être reconnue comme telle et mobilisée par les acteurs, implique une nécessaire réflexivité du praticien sur la portée, les potentialités d'agir recelées par les différentes habiletés acquises ou développées. D'où l'importance accordée dans la recherche-action à plusieurs temps de retour d'expérience des participants : une première explicitation du vécu est faite collectivement en groupe à la fin de la journée, puis un retour individuel écrit dans les semaines suivant la journée et, enfin, une réflexion $a$ posteriori, trois mois après la formation a été réalisée sur la forme d'un entretien qualitatif. L'analyse qualitative des écrits et des entretiens a aussi été confrontée aux observations et prises de notes des chercheurs qui étaient postés dans les différents ateliers. Ainsi, comme il visait à rendre perceptibles les conditions d'émergence du faire ensemble, le dispositif a permis de faire ressentir les différences entre 
coordination et coopération, puis de mettre en visibilité des compétences communicationnelles minorées ou impensées dans le travail de management (Lépine, Parent, 2014 ; Lépine, 2013).

\section{En quoi le dispositif Org'impro interroge-t-il autrement le rapport entre communication, projet collectif et organisations ?}

\subsection{Dépasser les modèles traditionnels}

L'équipe de conception du dispositif avait pour objectif de proposer des exercices favorisant un questionnement, par la pratique d'improvisation, des systèmes d'oppositions binaires tels que liberté individuelle $v s$ projet collectif, temps libre $v s$ temps contraint au travail, créativité $v s$ gestion, espace ouvert $v s$ fermé, efficacité $v s$ émotivité, contrôle $v s$ lâcher-prise. Il s'agissait également de proposer un dispositif pédagogique qui mette en questionnements les représentations des participants sur la place de la pensée structurée par le langage dans sa relation à l'action individuelle et collective. Dans un contexte pédagogique qui valorise le contrôle et la différentiation de soi et de son action par la pensée rationnelle, peut-on faire expérimenter une autre façon de penser et d'éprouver la place d'un individu dans le collectif ? Peut-on explorer d'autres imaginaires du rapport individu collectif?

Si ces questionnements semblent urgents, c'est que les modèles organisationnels implémentés dans les organisations ne se nourrissent que très peu des recherches en sciences de gestion et du management qui, depuis près de dix ans, remettent en cause ces systèmes épistémologiques et s'appuient, pour certains, sur l'improvisation pour penser le management de projets (Vera et Crossman, 2008). Dans la majorité des parcours universitaires français, les modèles enseignés n'interrogent pas (ou peu) la place de la communication non verbale dans l'organisation des dynamiques de coordination et de coopération. Quelques exemples peuvent illustrer le manque de complexité des systèmes de représentation du travail collectif et en particulier le rôle de la communication et du leader dans les modèles enseignés.

Selon la majorité des modèles, le leader doit argumenter et reformuler les attentes du groupe et ses compétences sont verbocentrées. Cette représentation du leader construit la double illusion que l'agir collectif peut être structuré par une éthique de la discussion qui évacue la question des émotions et que l'action est orientée par un seul leader. Cette vision, bien qu'aujourd'hui battue en brèche, demeure présente dans les imaginaires de l'efficacité organisationnelle: certains ouvrages anciens consacrés à la dynamique des groupes (voir Aubry et Saint-Arnaud, 1963, p. 34) comme de plus récents sur le management (Raynal, 1997, p. 226) voient les auteurs 
surdéterminer à la fois le rôle du langage et celui du leader en charge de la structuration du groupe par ses compétences en rhétorique. Plus récemment le design management (Borja de Mozota, 2002) qui s'appuie sur l'arbre du design a le mérite de souligner l'apport de la dimension esthétique dans les projets organisationnels, mais ne développe pas suffisamment les « racines de l'arbre » que sont les conditions d'émergence du faire ensemble - le sensible -, à savoir la partie cachée et peu connue de l'iceberg. Avec un autre vocabulaire, les travaux de Nonaka et Takauchi (1997) portent sur le processus de construction collective d'une connaissance créatrice. Si ces auteurs ont mis en exergue le rôle central des connaissances tacites dans les projets organisationnels, ils n'expliquent pas les mécanismes d'émergence et d'élaboration de ces connaissances.

Aussi, dans de nombreux ouvrages, cette grammaire de l'action reste toujours présente. Pour exemple, dans l'Encyclopédie des ressources humaines rééditée en 2012, Nathalie Raulet-Croset reprend ces différents courants de pensée, dont les thèses de K. Schmidt qui distingue trois formes de coopération : « la coopération d'amplification qui permet de dépasser les limites individuelles, la coopération de « diversification » qui amène chacun à se spécialiser, la coopération de confrontation qui permet la confrontation des points de vue»(1994). Elle reprend également celles de M. Grosjean et de M. Lacoste (1999) qui se sont en particulier intéressées au rôle du langage dans les coopérations, et notamment à l'existence d'implicite dans les situations de coopération. Dans les termes de Raulet-Croset, « une part de la coopération repose en effet sur du non-dit, car les règles qui la fondent sont partagées, et connues par les différents participants » (Borzeix, 1994, repris par Raulet-Croset). Pour les analyses de la coopération, il s'agit de prendre en compte également cette distinction entre implicite et explicite soit pour étudier l'implicite dans la coopération, soit pour mieux comprendre le rôle d'une explicitation volontaire de savoirs ou de référentiels communs en tant que supports à la coopération.

L'ensemble de ces modèles n'intègre pas les processus cognitifs que sont l'enaction et l'échoïsation qui sont pourtant essentiels dans la coopération. L'enaction est un concept développé par Francisco Varela (repris par F. MartinJuchat, 2008, p. 54) afin de signifier que tout contenu cognitif est la partie émergente d'un processus enraciné dans le corps. Les contenus cognitifs sont des images issues d'empreintes somatiques qui résultent non seulement d'une expérience personnelle, mais aussi de son rapport d'influence corporelle au monde et aux autres. Or, de tels éléments ont été perçus par les participants d'Org'impro, ainsi, tel qu'il l'exprime avec ses mots, cet étudiant qui prend conscience des liens étroits entre la pensée et le corps - sans toutefois parvenir à sortir d'une forme de dichotomie :

Il y a trois types de personnalité qui rentrent dans l'espace : certains qui agissent et qui réfléchissent et ceux qui font les deux à la fois - et ceux qui réfléchissent et 
ensuite qui agissent. (...) Ça a changé ma manière d'être : moins dans la réflexion et plus dans l'action - je suis trop dans la réflexion.

L'échoïsation, concept proposé par J. Cosnier pour désigner la capacité instinctive des corps à s'imiter par un processus de mimisme non conscient (repris par MartinJuchat, 2008, p. 50), apparaît fondamentale durant la période de développement de l'enfant et également à l'âge adulte même sans être accompagné de conscience. D'autres participants ont attesté avoir ressenti un tel phénomène corporel, de même qu'ils ont perçu certains facteurs de différenciation susceptibles de faire émerger des leaderships variables au gré des compositions :

Je pense à l'exercice où l'on était tous en ligne et marchait en même temps au même rythme, plus ou moins rapide. Cet exercice faisait rapidement apparaître les personnes qui donnaient le rythme et qui apparaissaient donc comme les « leaders » ainsi que les «suiveurs» qui accompagnaient tout simplement le rythme».

Ces processus sont essentiels, si l'on s'appuie sur la thèse de Zarifian (1996) concernant la coopération. Selon Zarifian, un événement ou un incident peuvent déclencher une coopération perçue comme nécessaire et associée à une volonté de réactivité et d'adaptabilité. À ce titre, la démarche pédagogique a consisté, après avoir proposé des outils, à créer artificiellement une situation de rupture destinée à conduire les étudiants à coopérer. En effet, l'engagement comme la prise de risques sont peu familiers à des étudiants plus coutumiers de la situation anonyme et passive propre au cours magistral. La mise à l'épreuve de performances observées par d'autres a favorisé la bascule du connu vers l'inconnu, ainsi que le consentement à évoluer dans celui-ci en co-construisant une action collective.

\subsection{Expérimenter corporellement la construction d'une action collective}

Comment concevoir à l'Université des dispositifs pédagogiques du faire ensemble qui préparent à appréhender individuellement et collectivement des effets non anticipés de l'action sociale finalisée ? Comment faire ressentir un espace-temps non linéaire dans des groupes projets, à partir d'imaginaires individuels plus à l'aise avec des modèles d'action issus de la pensée rationnelle? Aussi, comment préparer les futurs professionnels à saisir le rôle du hasard, de l'aléa, de l'inattendu, de l'imprévu dans les processus collectifs ? Comment les préparer à appréhender le rôle des émotions dans les groupes? À gérer les conflits dans des prises de leadership non prévues et non prévisibles ? Peut-on les entraîner à écouter ce qui émerge d'un collectif par échoïsation ? Peut-on les aider à imaginer un partage du leadership ? Sans prétendre répondre à l'ensemble de ces enjeux cruciaux, Org'impro a vocation 
à initier un «bousculement» à la fois physique et mental des cadres habituels, à engager un questionnement nouveau comme l'illustre le témoignage suivant:

Le but de l'atelier était effectivement de tester des comportements hors des cadres de vie des étudiants, et je pense que l'ensemble a été un franc succès ! (...). Le fait de prendre le temps, de revenir à l'essentiel de la nature de l'homme, c'est-à-dire les relations avec autrui, et la relation avec soi-même, a permis de se comporter de façon inhabituelle. Ce constat met en exergue un point important de notre société actuelle : si tout est mouvement perpétuel, quelle position devonsnous adopter pour comprendre l'autre ? Devons-nous être en mouvement avec lui ? Ou devons-nous au contraire nous défaire de ce mouvement pour mieux le comprendre?

L'improvisation comme pratique collective et corporelle du mouvement procure pour l'individu des étonnements: comme de se découvrir des compétences de leadership ; de ressentir un possible entre-deux, tel un interstice ouvert entre des mouvements « rationnels» (planification, argumentation) et d'autres nommés « irrationnels » (enaction, intuition). Dans cet entre-deux, stimulant la créativité, se construit pourtant une part du sens nécessaire à l'action. Fournir à l'individu la possibilité, dans un groupe, de ressentir les possibilités de circulation entre des mouvements engendrés par lui-même et par les autres est fondamental, car cela nourrit la coopération et autorise une vision renouvelée du leadership. Selon les termes d'un étudiant :

Au cour de l'improvisation, il y a aussi la co-construction. Avec l'organisation hiérarchique, c'est la logique du petit chef qui règne. L'utilisation de l'improvisation pourrait permettre aussi plus de co-construction entre les personnes. L'impro permet de prendre en compte la proposition de l'autre et faire avec, de créer à partir de ça sans la nier. »

«Ce travail doit permettre d'accepter de faire circuler le leadership... changer de rôle dans l'organisation.

Pour engager dans un groupe un processus coopératif, des processus psychoaffectifs sont nécessaires quoique peu visibles. Org'impro souligne que l'improvisation se situe précisément sur les plans de la présence et de la co-présence physiques, puis de l'engagement corporel, interactionnel et aussi affectif. Il est important de partager des émotions afin de développer la confiance dans le groupe ; dans la plupart des situations de groupe, l'engagement des individus œuvre à la conformité dans le but de créer et de préserver la cohésion du groupe et de la (re)production de normes communes (Oberlé \& Beauvois, 1995). Des interactions vécues surgissent les règles du faire ensemble, les valeurs partagées, le langage commun du projet qui prend forme. Cette étape de consolidation collective est intéressante à observer, car elle procède en différents moments. L'échoïsation ou mimétisme interactionnel apparaît quand les membres d'un groupe composent entre 
eux, se synchronisent, s'accordent et, enfin, coproduisent des mouvements et des signes qu'ils soient verbaux ou non verbaux (physiques, affectifs ou linguistiques). En se mettant en situation d'écoute pour composer avec les ressources en présence, ils activent, dans les termes d'un participant, leur capacité d'enaction :

Org'Impro révèle donc cette capacité à communiquer et à ériger un mouvement collectif par le biais du corps et des sensations : dans l'exercice des tableaux, il semblerait que le corps puisse faire sens par la simple présence d'un individu immobile. Il montre que nos décisions peuvent être portées par ces sensations et par celles des autres, nous immerge dans une ambiance collective et, surtout, que le corps n'est pas seulement porteur de langage au sens propre.

Aussi, le ressenti des participants confirme ce qu'Org'Impro visait à expérimenter, à savoir, faire éprouver aux individus présents une autre façon de vivre l'action collective et engendrer de la coopération via des séquences d'improvisation autour d'un événement :

J'ai découvert des compétences comme la réactivité des personnes sur le plan comportemental physique dans son espace à travers ces déplacements et aussi j'ai découvert que le respect avant tout est l'écoute du groupe.

Le résultat apparaît donc satisfaisant pour cette première étape exploratoire. En une unique journée, des groupes constitués d'individus de différents horizons et sans connaissance commune ont réussi à construire l'œuvre collective d'une composition improvisée, tout en ayant au préalable défini leur protocole de travail et leur style à partir de techniques acquises et rapidement réappropriées par le groupe. Cette phase d'activation de « compétences improvisationnelles » soumises au regard des autres a cependant été diversement vécue tant le regard d'autrui semble pensé comme évaluatif et critique : bien acceptée lorsqu'on est assuré d'un climat bienveillant, l'exposition de soi dans une situation moins contrôlée est également anxiogène.

\section{Quelques éléments de conclusion}

La recherche-action présentée n'est pas encore aboutie car une analyse plus fine des retours d'expérience auprès des participants apparaît nécessaire ; c'est l'objectif d'une seconde session destinée approfondir l'expérimentation (décembre 2015), dans laquelle il s'agira aussi d'examiner les possibilités de transfert des compétences mobilisées dans et par l'improvisation vers les pratiques et contextes professionnels variés. L'expérience sera donc renouvelée dans le cadre de la formation continue et des entretiens conduits après une période de retour des professionnels dans leurs organisations. Il n'est pas tant question de supposer que les pratiques évolueront radicalement à la suite d'une formation de ce type que d'examiner si le regard des 
participants s'est modifié et si des formes locales et diverses d'appropriation de l'expérience se dessinent.

Il faut le souligner, le risque d'une instrumentalisation de telles pratiques mobilisant les ressources de l'improvisation apparaît, à l'évidence, toujours présent. Toutefois, si une vision idéalisée car décontextualisée des enjeux de pouvoir et des agir stratégiques au travail serait illusoire, il ne s'agit pas avec Org'impro de promouvoir l'improvisation comme une technique parmi d'autres dans la boîte à outils des managers ou des communicants, mais a contrario d'élargir la conscience critique des participants en formation, de favoriser la réflexivité des professionnels, de faire toucher par l'expérience vécue la finesse, la richesse et la complexité et pourquoi ne pas le souligner également ? - le plaisir du faire ensemble.

Une telle ouverture émancipatrice se comprend sur différents plans. Tout d'abord au plan épistémologique il s'agit de rompre avec l'approche verbocentrée de la coopération et de réintroduire les dimensions corporelles et affectives nommées langages non verbaux dans les modèles de la pensée communicationnelle. Ensuite, au plan praxéologique, l'improvisation offre l'expérience de rapports humains qui bouleversent les codes de la proxémique et de la kinésique habituellement très contraints et codifiés dans nos organisations occidentales. Cette dimension conditionne elle-même l'accès à un plan qu'il convient de caractériser comme politique : dans ses conditions réelles, la prise de décision de chacun entre en écho avec celle des autres, et ce processus met en conscience l'action collective. La réflexivité sur l'action collective ainsi engendrée permet de s'arracher à des habitudes de comportement et à des schémas d'interaction politique fortement prégnants, corporellement très ancrés bien qu'invisibles et hérités de conceptualités anciennes (Ménissier, 2011), au profit de l'émergence de cette modalité de l'agir ensemble qu'Arendt nommait la «puissance » (power), identifiée comme source de conscience et d'émancipation (Arendt, 1958, chap. V). Enfin, au plan pédagogique, l'apprentissage de compétences relationnelles, esthésiques et esthétiques portées par l'improvisation nous paraît porteur d'une émancipation à l'égard des compétences dominantes issues de la gestion et des techniques de production des outils, dans les métiers de la communication notamment (Lépine, Martin-Juchat, Millet-Fourrier, 2014, Martin-Juchat, 2014).

\section{Bibliographie}

Ardouin, T., 2004, "Pour une épistémologie de la compétence", dans Astolfi, JP., Savoirs en action et acteurs de la formation, Rouen Le Havre: Publication université, pp 31-50.

Arendt, H., 1958, Condition de l'Homme moderne, trad. H. Fradier, in L'humaine condition, 2012, édition sous la dir. de Ph. Raynaud, Paris : Gallimard. 
176 Dynamiques de professionnalisation en communication

Aubry, J.-M., Saint-Armaud, Y., 1963, Dynamique des groupes, Paris : Éditions Universitaires.

Borja de Mozota, B., 2002, Design Management, Paris : Éditions d'organisation.

Baudouin J.-M., Friedrich, J., 2001, « Introduction. Théories de l'action et éducation », in J.-M. Baudouin \& J. Friedrich, (dir.), Théories de l'action et éducation, De Boeck Université, pp. 7-24.

Després, A., 2010, « Le Contact Improvisation : une situation particulière d'enseignement-apprentissage " in eJRIEPS, e-Journal de la Recherche sur l'Intervention en Éducation Physique et Sport, pp. 23-39.

Henriot, J., 1969, Le jeu, Paris : PUF.

De La Broise, P., 2013, « La lutte pour la reconnaissance ? code, chartes, référentiels et autres manifestes de professionnels français de la communication », Cahiers du RESIPROC $N^{\circ} 1$, pp. 33-50.

Lahire, B., Rosental, C., (dir.), 2008, La cognition au prisme des sciences sociales, Paris : Archives contemporaines Editions.

Lépine, V., Martin-Juchat, F., Millet-Fourrier, Ch. (dir). 2014, Acteurs de la communication des entreprises et des organisations, Pratiques et perspectives, Grenoble : PUG.

Lépine, V., Parent, B., 2014, « De la visibilité à l'individuation des cadres hospitaliers : l'obstacle de la dyschronie ", Communication \& Organisation, $\mathrm{N}^{\circ} 44$, pp.41-52.

Lépine, V. 2013, «Identité professionnelle et professionnalisation des cadres de santé ; le rôle des compétences communicationnelles », (pp. 177-188), dans Les communications organisationnelles. Comprendre, construire, observer, dir. P. Alemanno, S. et Parent, B., Paris : L'Harmattan.

Martin-Juchat, F., 2008, Le Corps et les médias : la chair éprouvée par les médias et les espaces sociaux. Bruxelles, Belgique : De Boeck.

Martin-Juchat, F., Zammouri, Z., 2013, «Expérimenter les relations entre artistes et scientifiques : l'appropriation de capteurs de mouvement par des danseurs », revue ESSACHESS - Méthodes expérimentales en communication, Volume 6, $n^{\circ}$ 1(11), URL : http://www.essachess.com/index.php/jcs/article/view/199

Martin-Juchat, F. 2014, « La dynamique de marchandisation de la communication affective ", Revue française des sciences de l'information et de la communication [En ligne], 5|2014, mis en ligne le 17 juillet 2014. URL : http://rfsic.revues.org/1012 
Mayen, G., 2012 «Qu'est-ce que la performance ? », Dossiers pédagogiques. Spectacles vivants et arts visuels du Centre Pompidou : en ligne.

Ménissier, T., 1999, «Ordini et tumulti selon Machiavel : la république dans l'histoire », Archives de Philosophie, avril-juin, tome 62-2, pp. 221-239.

Ménissier, T., 2003, " Philosophie politique et anthropologie de la férocité », Raisons politiques, $\mathrm{n}^{\circ} 9$, février, pp. 19-31.

Ménissier, T., 2011, La liberté des contemporains. Pourquoi il faut rénover la République, Grenoble : PUG.

Millar, S., 1968, La psychologie du jeu, Paris : Payot.

Nonaka, I. Takauchi, H. 1997, la connaissance créatrice. Dynamique de l'entreprise apprenante, Bruxelles : De Boeck Université.

Oberlé, D. et Beauvois, J.L., 1995, « cohésion et normativité », in Mugny, G., Oberlé, D., Beauvois, J.L, Relations humaines, groupes et influence sociale, Tome 1, Grenoble, Presses Universitaires de Grenoble : pp.75-90.

Raynal, S., 1996, Le management par projet, Paris, Les éditions d'organisation.

Raulet-Croset, N., 2012, «Coopération. La coopération au travail», in L'encyclopédie des Ressources humaines, Paris : Guilbert, pp. 320-326 (1ère édition 2003).

Schön, D., 1994, Le Praticien réflexif. À la recherche du savoir caché dans l'agir professionnel. Montréal : Éditions Logiques, (1ère ed. 1983).

Varela, F., Thompson, E., Rosch, E., 1991, The Embodied Mind: Cognitive Science and Human Experience, 1991, MIT Press (L'inscription corporelle de l'esprit : sciences cognitives et expérience humaine, trad. V. Havelange Paris, Editions du Seuil, 1996).

Vera D., Crossan, M., 2008, Improvisation and Innovative Performance in Teams Organization Science 16(3), pp. 203-224.

Vergnaud, G., 1990, La théorie des champs conceptuels. Recherche en didactique des mathématiques, 10, 2-3, pp. 133-170.

Walter, J., 1995, Directeur de la communication. Les avatars d'un modèle professionnel, Paris : L'Harmattan.

Winograd, T., 1987-1988, "A langage/action perspective on the design of cooperative work”, in Human-Computer Interaction 3 h 1 (1987-1988) : 3-30.

Winograd, T., Flores, F., 1986, Understanding Computer and Cognition : A new Foundation for Design, New Jersey : Ablex. 
178 Dynamiques de professionnalisation en communication

Wittorski, R., 2008, « La professionnalisation », Savoirs 2/2008 (n¹7), pp. 9-36.

Wittorski, R., 1998. De la fabrication des compétences. Éducation Permanente, 135, pp. $57-70$.

Zarifian P., 1996, Travail et communication - Essai sociologique sur le travail dans la grande entreprise industrielle, Paris : PUF.

Zarifian P., 2010, Le Travail et la compétence, entre puissance et contrôle, Paris : PUF. 